\title{
La Abducción como Estrategia Cognitiva para Potenciar el Nivel Inferencial en la Comprensión Textual
}

\section{Abduction as Strategy to Upgrade Inferential Level in Textual Comprehension}

\begin{abstract}
Claudia María Zapata Cano
PhD Ciencias de la Educación. Universidad Rafael Belloso Chacín. Docente de Secundaria Colegio Distrital Buenos Aires, CODIBA Coordinadora de Investigación Universidad Autónoma del Caribe, Colombia. Licenciada en Lengua Castellana, Universidad de Antioquia, Colombia Clauza2502@icloud.com
\end{abstract}

Para citar este artículo: Zapata, M. (2016). La Abducción Como Estrategia Cognitiva para Potenciar el Nivel Inferencial en la Comprensión Textual, Escenarios, 14 (1), p,p 63-71

DOI: http: / / dx.doi.org/10.15665/ esc.v14i1.878

Recibido: febrero 25 de 2016

Aceptado: marzo 31 de 2016

\section{RESUMEN}

El presente artículo es el resultado de la experiencia de investigación, basado en el desarrollo del pensamiento abductivo como estrategia para mejorar el nivel inferencial de la comprensión textual en los niños del grado cuarto de la básica primaria de la Institución Educativa Finca la Mesa del municipio de Medellín, Antioquia En el Colegio Cooperativo Juan del Corral se realizó la primera exploración en el tema de la abducción para mejorar la comprensión lectora con jóvenes del grado noveno de la básica secundaria; investigación que consistió en identificar de un lado, los niveles más bajos de desempeño en la comprensión textual de los estudiantes y por otro, aplicar los procedimientos de la hipótesis abductiva con base en los estudios de la teoría Peirceana para la resolución de cuentos enigmáticos favoreciendo cada uno de los niveles de pensamiento, especialmente el inferencial, potenciando además la capacidad creativa, argumentativa e investigativa del estudiante. El texto está especialmente dedicado a exponer los criterios y resultados, que desde la perspectiva Peirceana, puede generar aportes significativos en materia de Pedagogía, didáctica y métodos de enseñanza que potencien las habilidades mentales del educando.

Keywords: Education, Teaching, Teaching Strategies, Abduction, Hypothesis, Inference, Reading Comprehension

\begin{abstract}
This articles is the result of researching experience based on abductive thinking development as strategy of improvement in inferential level of textual comprehension in fourth grade students of elementary school from Institución Educativa Finca la Mesa municipality of Medellin, Antioquia; this research consist of identify low level of performance in textual comprehension from students, on the other hand, apply procedures of abductive hypothesis based on studies of Peirce theory for resolution of enigmatic stories favoring each of thinking levels primarily the inferential one. In addition, promoting students`creating, reasoning and researching ability. The text is specially dedicated to show criteria and results, which from Peirce perspective, can generate meaningful contributions in a matter of Teaching, didactic, teaching method that strengthen thinking abilities on learner.
\end{abstract}

Palabras Clave: Educación, pedagogía, estrategias de enseñanza, abducción, hipótesis, inferencia, comprensión de lectura. 


\section{INTRODUCCIÓN}

Considerar la efectividad de un programa experimental como método de enseñanza para mejorar el nivel inferencial en la comprensión textual en niños (as) del grado cuarto de la Básica Primaria en poblaciones vulnerables de pobreza económica y violencia intrafamiliar y social en el municipio de Medellín, fue la intención principal que impulsó el estudio, diseño, la ejecución y evaluación del presente trabajo investigativo.

Pese a lo anterior y de acuerdo con las políticas educativas Nacionales de Colombia, los estudiantes de la básica no superan la exigencia mínima que se espera en Lenguaje, incluso en el contexto latinoamericano, los estudiantes arrojan mejores resultados que Colombia (ICFES, 2008,2010; OECD, 2008, 2009). Estas políticas se encuentran enmarcadas en el Plan Decenal de Educación 2016-2025, en el título III: El derecho a la educación y la calidad de la educación; la cual se espera que los colegios superen el promedio del país. En el caso de Lenguaje es de 259 sobre 500 , e ir aumentando cada año por encima de ese promedio según el ritmo académico de cada Institución educativa. Recuperado el 21 de Julio de 2016 de www.mineducacion.gov.co/1621/ articles-312490_archivo_pdf_plan_decenal.pdf Programa "Todos a Aprender" para la transformación de la calidad educativa. Guía $N^{\circ} 1$. Pág.4 Ante esta problemática, se realizó una investigación de diseño cuasi experimental, basado en el empleo de variables independientes: lógica abductiva, realizado bajo el enfoque de innovación pedagógica, currículo y didáctica y la observación de sus efectos en la variable dependiente: Comprensión de lectura en su nivel inferencial desde el pensamiento y lógica textual. La aplicación del pretest y postest fueron con grupos intactos (Pantoja, A. 2009).

El trabajo investigativo, estuvo centrado en comprobar desde la teoría Persiana, la posibilidad de potenciar en mayor escala la lógica inferencial, para el mejoramiento de la comprensión de lectura, teniendo para tal efecto y como punto de partida, la generación de pequeñas hipótesis que los estudiantes produjeron y a la que se definió, de acuerdo al teórico Charles Sanders Peirce como "abducción"; término usado durante la puesta en marcha de la estrategia didáctica; logrando, a través de resultados observables, la presentación de algunos aportes significativos en materia pedagógica, enfocados a la didáctica y métodos de enseñanza, para el desarrollo de habilidades de pensamiento lógico inferencial en el educando.

La puesta en marcha metodológica del estudio cuasi experimental, se llevó a cabo en cuatro momentos: en el primero titulado Reconocimiento, tuvo como fin el reconocimiento de la población experimental en su contexto. En el segundo llamado Asimilación, se ambientó y preparó al grupo experimental sobre el método de razonamiento lógico e inferencial abductivo, mediante de la observación de varios videos detectivescos o policiacos en donde se aplicó la lógica abductiva para resolver los casos o misterios de la historia. El análisis introspectivo de cómo el detective resuelve los enigmas, fueron la base sobre la cual los estudiantes desarrollaron la lógica inferencial para el razonamiento lógico. El tercer momento designado como Afianzamiento, tuvo como propósito el afianzamiento de la lógica o pensamiento abductivo en el grupo experimental mediante el uso de talleres y fragmentos literarios, en especial, cuentos de Arthur ConnanDoyle, con los cuales, los estudiantes generaban sus propias hipótesis como un intento por resolver los casos enigmáticos de la historia.

Finalmente, se realizó un último momento denominado Constatación; en donde se analizó, verificó y describió los hallazgos; alcances e impacto de la aplicación de la lógica abductiva en el mejoramiento de la comprensión de lectura en los educandos.

¿Por qué la abducción para potenciar el nivel inferencial de la comprensión textual? ${ }^{1}$

La comprensión textual es entendida como un proceso cognitivo en el que el lector construye

1 Este epígrafe se ha ido enriqueciendo de los estudios sobre la abducción en la comprensión lectora, adelantados por la autora del presente artículo en sus estudios de pregrado en la licenciatura de educación Básica con énfasis en Lengua Castellana de la Universidad de Antioquia, publicado en Zapata. C. (2010). La abducción creativa como recurso para mejorar la comprensión textual. Recuperado el 11 de Julio de 2016 de file: / / C: / Documents\%20and\%20 Settings / XP /Mis\%20documentos/Downloads/IVJornadasArgentinaZapata\%20(1).pdf 
una interpretación de esa información teniendo en cuenta las pistas que éste ofrece y sus conocimientos previos (Parodi citado en Hurtado, Sossa y Arango, 2008). De lo anterior, se puede inferir que la comprensión lectora se efectúa mediante el desarrollo de habilidades mentales que no surgen de manera espontánea, sino que parte unos conocimientos previos adquiridos por el sujeto a lo largo de su vida y de ciertas marcas textuales o información que ofrece el texto. Desde este punto de vista se extrajeron dos variables independientes tales como improntas (pistas o información clave del texto) y reglas (conocimientos previos).

Ahora bien, de acuerdo con McNamara, existen varios niveles de comprensión lectora: local, global, elaborada y critica, de las cuales, sólo para este estudio nos enfocaremos en la lectura elaborada. Según el autor, en este nivel el lector va más allá de la lectura local, ya que realiza inferencias, resuelve problemas, proponiendo alternativas de solución y reestructura su conocimiento previo (McNamara 2004, citado por Mateos 2009). Esto implica lanzar conjeturas yconstruir hipótesis generando nuevas ideas; partiendo de las ideas explícitas del texto.

En consonancia con lo anterior, Nubiola (CP 3, 2001) en sus traducciones hechas al castellano de Charles Sanders Peirce, acerca de los estudios que éste adelantó sobre el desarrollo de la lógica, precisa que "la inferencia es un procedimiento en el cual el individuo formula juicios, que a su vez son determinados por otros juicios, conducentes a un conocimiento final en el que se espera que repose el pensamiento sin dar lugar a la duda (Zapata, 2010, p. 3, citando a Nubiola, 2001).

Así pues, la abducción reconocida como un ejercicio inferencial requiere la formulación de conjeturas a partir de reglas o conocimientos previos y reordenarlas en función de la causalidad en que se presentan, asociar las premisas para finalmente crear sus posibles hipótesis a los enigmas planteados (Mejía, 2004) ; en otras palabras la abducción posibilita todo un juego de habilidades cognitivas necesarias para la inferencia y la comprensión lectora en general.
Se espera con este trabajo investigativo no sólo dinamizar la lógica, la comprensión, el análisis y la inferencia en los estudiantes sino aportar un método de enseñanza poco usual en la didáctica del lenguaje y lógica textual.

\section{Método}

Aunque la investigación fue mixta, alternando la metodología cualitativa en forma de registros de clase y la cuantitativa a través de la aplicación de pruebas no paramétricas SPSS, por ello se hará referencia para este artículo a los resultados y análisis cuantitativos, sin dejar de lado aspectos cualitativos indispensables, en especial en la propuesta o tratamiento y en la discusión de resultados.

La investigación se realizó con estudiantes del grado cuarto de la básica primaria de la Institución Educativa Finca la Mesa del municipio de Medellín, conformado por cincuenta y cuatro (54) estudiantes de los cuales hay treinta y tres (33) niños y veintiún (21) niñas, con edades que oscilan entre los nueve y los diez años.

La muestra representativa se tomó bajo la técnica del Muestreo Aleatorio Estratificado (MAE), sobre la tercera parte de la población teniendo como criterio de referencia, estudiantes que representen a toda la población, y en la misma proporciónen edad, género y resultados cognitivos, tanto en desempeños altos, medios y bajos, según arrojó los diferentes niveles de desempeño en la prueba diagnóstica de comprensión lectora. (Pantoja, 2009)

Una vez identificado el planteamiento del problema, es decir, el bajo nivel de comprensión lectora en los estudiantes del grado cuarto de la básica primaria, especialmente en el nivel de inferencia; se formuló la siguiente pregunta problematizadora: ¿Es plausible, a través del desarrollo del razonamiento abductivo, potenciar la comprensión lectora desde el pensamiento y lógica textual mejorando la capacidad inferencial en los estudiantes del grado cuarto de la básica primaria de la Institución Educativa Finca la Mesa del municipio de Medellín?. A partir de ahí, se realizaron los estudios teóricos, referenciales y legales que sustentaban la propuesta de 
investigación; en especial del científico Charles Sanders Peirce ${ }^{2}$. Posteriormente se procedió a la ejecución de la misma. A continuación la investigación se llevó a cabo en cuatro momentos:

El primero titulado Reconocimiento, tuvo como finalidad la exploración de la población experimental en su contexto social, familiar, económico, cultural y académico; para lo cual se les aplicó una encuesta socio demográfica, posteriormente analizada en la escala SPSS.

Para la inspección del nivel académico se realizó un pre test utilizando la comprensión de lectura con preguntas cerradas conteniendo los tres niveles de comprensión que evalúa el ICFES: literal, Paráfrasis e inferencia. (2009, p. 11) ${ }^{3}$

En el segundo momento llamado Asimilación, se enseñó al grupo experimental, el método de razonamiento lógico e inferencial abductivo, mediante de la observación de videos detectivescos o policiacos en los que se aplicó la lógica abductiva para resolver casos.El análisis introspectivo y retroalimentación de cómo el detective resuelve los enigmas, fueron la base cognitiva sobre la cual los estudiantes desarrollaron la lógica inferencial para el razonamiento lógico desarrollado. Se realizó una exploración de las pistas (información) o improntas que el texto ofrecía. Las cuales eran sometidas unas sospechas iniciales y posteriormente a sacar conjeturas argumentadas bajo el fundamento de la regla (conocimientos previos) para finalmente, el sujeto en estudio, lanzar su hipótesis abductiva del interrogante por resolver. Se aceptaban todas las hipótesis posibles siempre y cuando éstas se desprendieran de una conjetura de acuerdo a la pista hallada fundamentada en una regla que sustente su teoría, llevando así una secuencia lógica, una reordenación de las ideas en el razonamiento abductivo.

El tercer momento designado como Afianzamiento, tuvo como propósito el asentamiento de la lógica o pensamiento abductivo en los educandos mediante talleres de fragmentos literarios con el fin de proponer hipótesis a situaciones enigmáticas o problemáticas dadas, bajo los parámetros antes descritos: improntas (o marcas textuales), premisas, conjeturas, regla e hipótesis resultante.

Finalmente, se realizó un último momento llamado Constatación en donde aplicó una postest de comprensión lectora comparada con la prueba inicial diagnóstica con el uso de la prueba no paramétricas, en donde se analizó, verificó y describió los hallazgos, alcances e impacto de la aplicación de la lógica abductiva en el mejoramiento del nivel inferencial en la comprensión de lectura en los educandos.

- Procedimientos utilizados para satisfacer los criterios técnicos de validez y confiabilidad de la prueba de variable dependiente.

En la fase 1 llamada identificación, se le aplicó a los estudiantes tanto del grupo experimental como del grupo control dos test de comprensión lectora con tres preguntas de selección múltiple, tendientes a evidenciar cual es el nivel de análisis en que se ubicaría tanto cada estudiante, como el grupo en general.

Tabla 1.Síntesis del diseño cuasiexperimental ejecutado

\begin{tabular}{|l|l|l|l|}
\hline Grupo & Pretest & $\begin{array}{l}\text { Variable } \\
\text { Independiente }\end{array}$ & Postest \\
\hline E & Y1 & $\mathrm{X}$ & Y2 \\
\hline C & Y1 & & Y2 \\
\hline
\end{tabular}

Fuente: Elaboración propia

\section{E: grupo experimental \\ C: grupo control}

Y1: Comprensión de lectura en su nivel inferencial desde el pensamiento y lógica textual antes de la intervención pedagógica

\footnotetext{
2 Algunos de los referentes conceptuales se pueden encontrar en Barrena, Sara. "Pragmatismo y Educación". Charles Sanders Peirce y John Dewey en las aulas. Editorial Machado Libros, Madrid, 2015 
X: intervención pedagógica con la lógica abductiva, realizado bajo el enfoque de innovación pedagógica, currículo y didáctica

Y2: Comprensión de lectura en su nivel inferencial desde el pensamiento y lógica textual después de la intervención pedagógica

Tabla 2. Sistema de variables

\begin{tabular}{|l|l|}
\hline independiente & $\begin{array}{l}\text { lógica abductiva, realizado bajo el enfoque de innovación } \\
\text { pedagógica, currículo y didáctica ( Improntas, Conjeturas y } \\
\text { Reglas) }\end{array}$ \\
\hline dependiente & $\begin{array}{l}\text { Comprensión de lectura en su nivel inferencial desde el } \\
\text { pensamiento y lógica textual . }\end{array}$ \\
\hline
\end{tabular}

Fuente: elaboración propia

Tanto para el pre test y el pos test se calculó la diferencia promedio en ambos grupos y luego las diferencias halladas en cada grupo se le aplicó la prueba de hipótesis no paramétricas, la cual nos mostró si la propuesta de intervención pedagógica produce un cambio significativo en los estudiantes.

Para la aplicación de la propuesta didáctica para mejorar el nivel inferencial de comprensión textual, se realizaron diez talleres de ejercicios con el razonamiento abductivo, y pruebas piloto aplicadas a estudiantes de cuarto grado de la Institución Educativa Finca la Mesa

Para garantizar la validación interna se tuvo la prueba piloto y los ajustes que de ella se desprendieron para la aplicación del pre test y el pos test, en torno a la maduración de la prueba.

Para el tratamiento o implementación de la propuesta, se tuvo en cuenta el criterio de un experto, el cual validó la confiabilidad y validez de los talleres que se aplicaron.

Para la validación externa se utilizó un muestreo aleatorio estratificado para garantizar la representatividad de la muestra. En cuanto a las fuentes de invalidación que pudieron estar presente, se tuvo en cuenta, el bajo nivel de lecto escritura, ya que se requiere mínimamente que el estudiante sepa leer literalmente y escribir con letra legible para que pueda responder a las preguntas y ejercicios propuestos sin necesidad de una ayuda tercera, sino que sea auto administrada por el integrante del grupo experimental. Este fue un criterio para tener en cuenta para la selección de la muestra.

\section{Resultados y Análisis}

Para el análisis de los resultados a nivel cuantitativo se utilizó la prueba no paramétricas SPSSStatistics versión 20, en la cual se compararon los puntajes del pretest y del postest para calcular su diferencia, posteriormente se emparejaron esas diferencias del grupo experimental y del grupo control para visualizar sus resultados:

Hipótesis inicial: SI existen diferencias significativas entre la prueba de pretest y postest del grupo experimental con relación al pensamiento y lógica textual para la comprensión lectora en su nivel inferencial de los estudiantes de cuarto grado de la básica primaria

Hipótesis nula: No existen diferencias significativas entre la prueba de pretest y postest del grupo experimental con relación al pensamiento y lógica textual para la comprensión lectora en su nivel inferencial de los estudiantes de cuarto grado de la básica primaria.

Nivel de significación: 0.05

De acuerdo a lo anterior, el nivel de significación asintótica fue 0,00 inferior a 0,05, rechazándose así la hipótesis Nula, lo cual indica que la abducción potencia el pensamiento y lógica textual mejorando la capacidad inferencial en los estudiantes del grado cuarto de la básica primaria de la Institución Educativa Finca la Mesa del municipio de Medellín.

Lo anterior se visualiza en las siguientes Graficas 1 y 2

El análisis de los resultados de Tabla 1 Gráfica 3 muestran que en el grupo experimental se dieron mejores resultados en los datos en el postest en comparación con el test inicial (promedio de $2,4)$ con un rango de datos igual para el pretest como para el postest y con una mayor desvia- 
Tabla 3.Grupo experimental 18 Estudiantes Del Grado $4^{\circ}$

\begin{tabular}{|l|c|c|c|c|c|c|c|c|c|}
\hline sujeto & $\mathbf{1}$ & $\mathbf{2}$ & $\mathbf{3}$ & $\mathbf{4}$ & $\mathbf{5}$ & $\mathbf{6}$ & $\mathbf{7}$ & $\mathbf{8}$ & $\mathbf{9}$ \\
\hline Pretest & 0 & 1 & 1 & 2 & 1 & 2 & 1 & 1 & 2 \\
\hline Postest & 3 & 3 & 2 & 3 & 2 & 3 & 1 & 1 & 3 \\
\hline Sujeto & $\mathbf{1 0}$ & $\mathbf{1 1}$ & $\mathbf{1 2}$ & $\mathbf{1 3}$ & $\mathbf{1 4}$ & $\mathbf{1 5}$ & $\mathbf{1 6}$ & $\mathbf{1 7}$ & $\mathbf{1 8}$ \\
\hline Pretest & 2 & 1 & 0 & 1 & 2 & 0 & 0 & 1 & 2 \\
\hline Postest & 3 & 3 & 3 & 3 & 3 & 2 & 1 & 1 & 3 \\
\hline
\end{tabular}

Tabla 4. Grupo control: 18 estudiantes del grado $4^{\circ}$

\begin{tabular}{l|c|c|c|c|c|c|c|c|c|}
\hline sujeto & $\mathbf{1}$ & $\mathbf{2}$ & $\mathbf{3}$ & $\mathbf{4}$ & $\mathbf{5}$ & $\mathbf{6}$ & $\mathbf{7}$ & $\mathbf{8}$ & $\mathbf{9}$ \\
\hline Pretest & 1 & 0 & 1 & 1 & 1 & 1 & 0 & 1 & 0 \\
\hline Postest & 1 & 0 & 0 & 1 & 1 & 1 & 0 & 0 & 0 \\
\hline Sujeto & $\mathbf{1 0}$ & $\mathbf{1 1}$ & $\mathbf{1 2}$ & $\mathbf{1 3}$ & $\mathbf{1 4}$ & $\mathbf{1 5}$ & $\mathbf{1 6}$ & $\mathbf{1 7}$ & $\mathbf{1 8}$ \\
\hline Pretest & 2 & 2 & 1 & 1 & 0 & 0 & 2 & 1 & 1 \\
\hline Postest & 0 & 1 & 1 & 0 & 0 & 0 & 0 & 0 & 0 \\
\hline
\end{tabular}

Tabla 5.Prueba de rangos con signo de Wilconson

\begin{tabular}{|ll|r|r|r|}
\hline & \multicolumn{1}{|c|}{ Rangos } \\
\hline & Rangos negativos & $0^{\mathrm{a}}$ & \multicolumn{1}{c|}{$\begin{array}{c}\text { Rango } \\
\text { promedio }\end{array}$} & $\begin{array}{c}\text { Suma de } \\
\text { rangos }\end{array}$ \\
\hline postest - pretest & $\begin{array}{r}\text { Rangos positivos } \\
\text { Empates }\end{array}$ & $15^{\mathrm{b}}$ & 8,00 & 120,00 \\
& $3^{\mathrm{c}}$ & & \\
& Total & 18 & & \\
\hline
\end{tabular}
a. postest $<$ pretest
b. postest $>$ pretest
c. postest $=$ pretest

Tabla 6.Estadísticos de contraste de las pruebas

Estadísticos de contraste ${ }^{a}$

\begin{tabular}{|l|r|}
\hline & $\begin{array}{c}\text { postest - } \\
\text { pretest }\end{array}$ \\
\hline $\begin{array}{l}\text { Z } \\
\text { Sig. asintót. } \\
\text { (bilateral) }\end{array}$ & $-3,502^{\circ}$ \\
\hline
\end{tabular}

a. Prueba de los rangos con signo de Wilcoxon b. Basado en los rangos negativos. ción estándar comparada con los test del grupo control. Los resultados de las dos pruebas del grupo control presentaron menor desviación estándar (variación) y unos resultados menores comparados con los resultados del grupo experimental.

Así, se observa en los resultados iníciales y finales del grupo experimental, después de la intervención pedagógica con la lógica abductiva, realizado bajo el enfoque de innovación pedagógica, currículo y didáctica, un mejor resultado 
en el nivel inferencial desde el pensamiento y lógica textual de las pruebas de comprensión de lectura.

\section{- Cualitativa}

Uno de los hallazgos que se evidenció en el ciclo de la educación primaria, además de la viabilidad de la enseñanza del razonamiento abductivo, fue la aplicación continua y sistemática del mismo: al inicio de la propuesta experimental, el ejercicio abductivo se realizaba cada una vez cada quince días. Este lapso de tiempo fue muy poco favorable ya que mientras la mente se adecuaba a esta nueva manera de razonar, es decir, bajo la inferencia que precede al desarrollo de la hipótesis abductiva, y al momento de volver a aplicar un nuevo ejercicio, se les olvidaba todo el procedimiento a practicado, volviendo de nuevo a iniciar el proceso cognitivo, retroalimentado El ejercicio entonces se intensificó una vez por semana y los avances se empezaron a notar. Al respecto Desde la perspectiva Peirceana, Barrena expresa que el ser humano "es un manojo de hábitos" . En consonancia con lo anterior, Peirce define "la materia" como "mente envuelta en hábitos" (CP1 5.57). Concluyo de lo anterior que, en todo aprendizaje sea cual sea (conceptos, procedimientos, costumbres) se debe crear el hábito, basado en la repetición, sistematicidad e intensidad; de acuerdo con el ritmo de aprendizaje que se desea implementar.

Otro hallazgo observado se presentó en el desarrollo del segundo momento de la estrategia pedagógica a la que se denominó "asimilación". Ésta se aplicó utilizando fragmentos de textos literarios de cuentos policiacos o enigmáticos en donde el grupo experimental, aplicando todas las pautas para el desarrollo de la abducción, resolvían los casos lanzando sus hipótesis. Este ejercicio favoreció mucho el razonamiento en la medida en la que ellos se involucraban con el personaje del detective y se sentían protagonistas de la historia.

Esto permitió potenciar la capacidad imaginativa de los estudiantes. Al respecto, Barrena menciona que, de acuerdo a las ideas de Peirce, "la imaginación no es una facultad separada que de vez en cuando utilizamos para nuestras fantasías, sino que esta entremezclada en todo lo que hacemos o pensamos, que es influida por nuestros pensamientos y acciones y que influye decisivamente en ellos" (2007, p. 105) de lo anterior concluyo que, en definitiva, la imaginación ocupa un papel relevante en el pensamiento, la motivación, la lógica y razonamiento del ser humano.

Ahora, la abducción, proporciona una presunción, una conjetura explicativa, una novedad. Pese a lo anterior, este razonamiento "no sería posible sin conocimientos previos" (Barrena, 2007, p.81) Con el grupo experimental este enunciado fue visible: en algunos casos puntuales de estudiantes, no se observó una mejoría significativa. Esta situación, me llevó a examinarlos con mayor detalle y se encontró que, en la mayoría

Gráfico 1. Excel basado en los resultados del pretest y postest grupo experimental

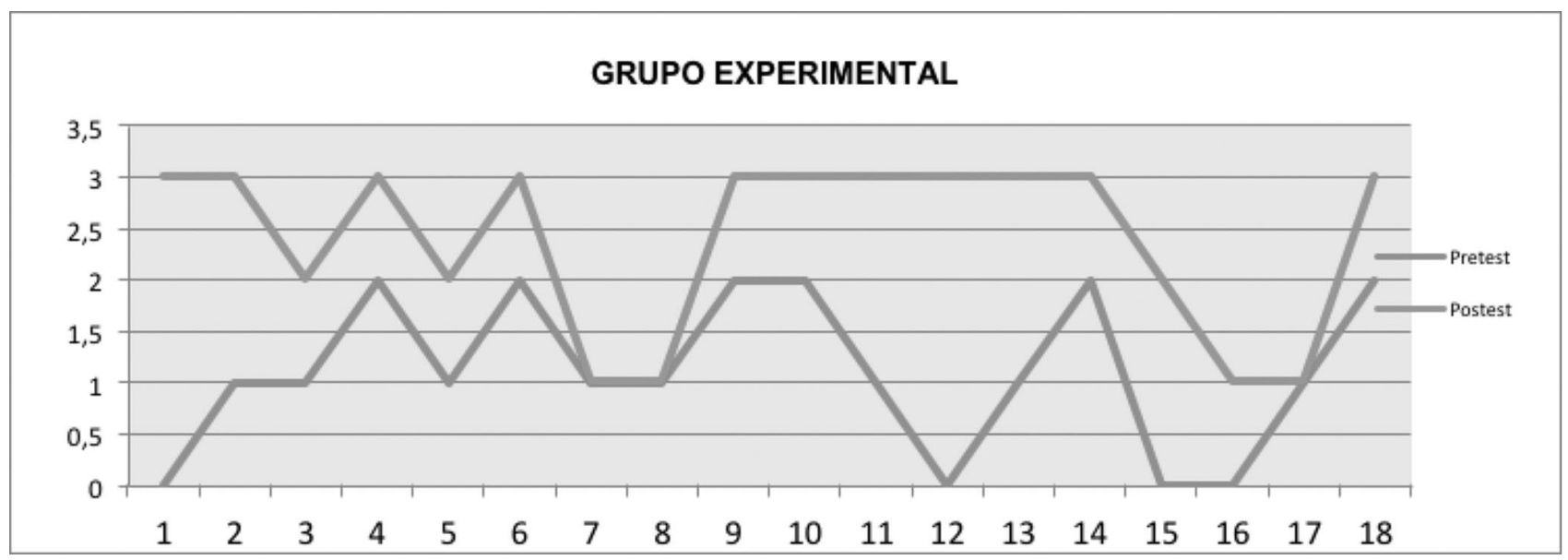


Gráfico 2: Excel basado en los resultados del pretest y postest grupo control

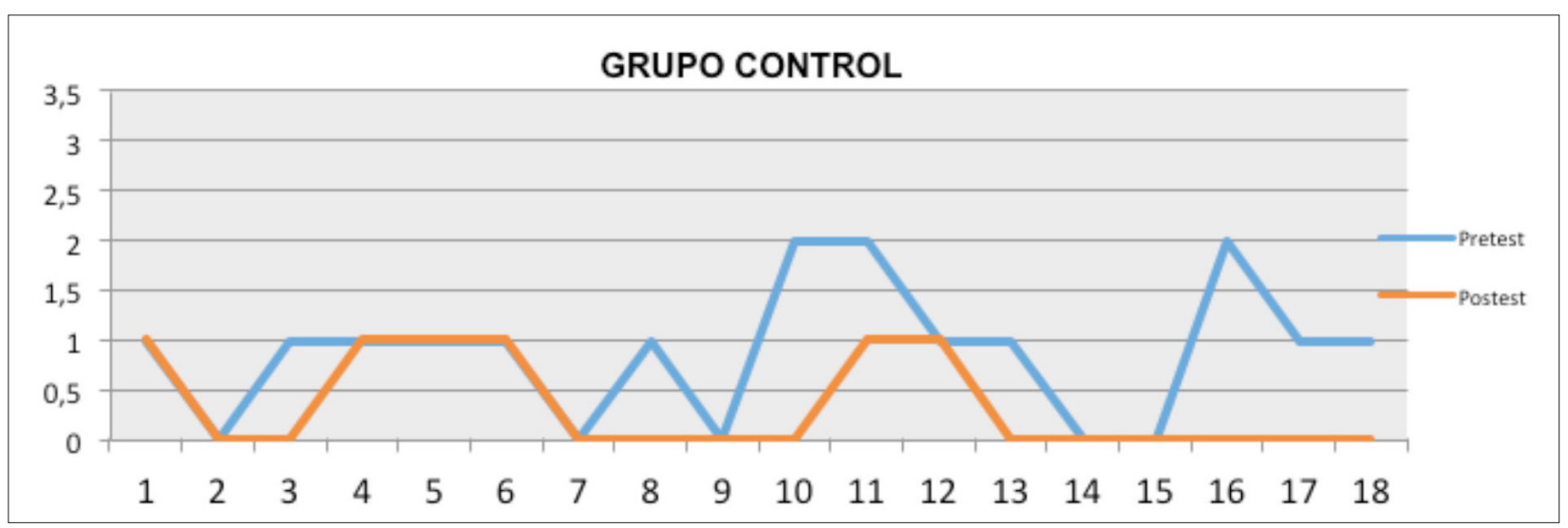

Tabla 1 Estadístico de contraste de los prueba de postest de los dos grupos

\begin{tabular}{|c|c|c|c|c|c|c|c|}
\hline Grupo & & $\mathrm{N}$ & Promedio & Desviación Estándar & Min & Max & Rango \\
\hline Experimental & Pretest & 18 & 1,111 & 0,758 & 0 & 2 & 2 \\
\hline & Postest & 18 & 2,389 & 0,850 & 1 & 3 & 2 \\
\hline Grupo & & $\mathrm{N}$ & Promedio & Desviación Estándar & Min & Max & Rango \\
\hline control & Pretest & 18 & 0,889 & 0,676 & 0 & 2 & 2 \\
\hline & Postest & 18 & 0,333 & 0,485 & 0 & 1 & 1 \\
\hline
\end{tabular}

Fuente: elaboración propia

Grafico 3 comparativo en la prueba postest del grupo experimental vs grupo control

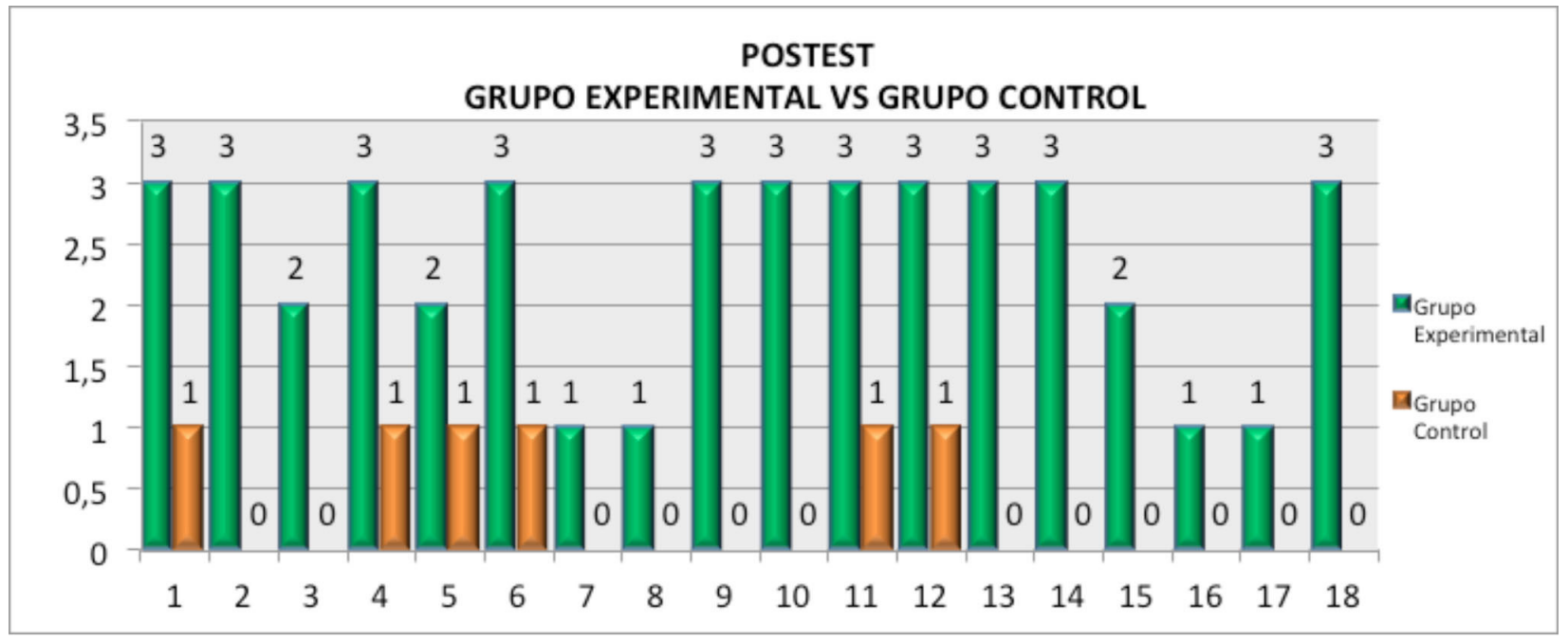

de los casos estos chicos tenían muy poco interés por la lectura de prensa, de historia, de cultura general; que al momento de conjeturar, sus hipótesis explicativas eran muy rudimentarias o incluso casi nulas. En palabras textuales de ellos "no se les venía nada a la mente" ninguna suposición o sospecha al respecto de determinada situación. Aun así, aunque sus conclusiones predictivas no tuvieron la mayor fuerza explicativa del hecho o fenómeno que se les planteaba, 
aprendieron el procedimiento para razonar inferencialmente.

\section{Conclusiones}

Finalmente, en el trabajo experimental, uno de los mayores aciertos, fue el mejoramiento de la capacidad de observación que desarrollaron un alto porcentaje de estudiantes. La observación tiene su relevancia en tanto que, a partir de ella se pone nuestros sentidos de observación y posteriormente, nuestro razonamiento en contacto con el mundo exterior, como punto de partida para indagar, plantear, discutir y concluir sobre alguna inquietud que ronda nuestra mente, tal como surge el pensamiento investigativo en el individuo.

Se ha estimado con efectividad que la abducción, que es, el tercer método de inferencia después de la deducción y la inducción; un ejercicio asertivo e innovador en niños como estrategia didáctica y metodológica en el proceso de enseñanza aprendizaje para el desarrollo de la inferencia en la comprensión textual.

Este trabajo abre otras posibilidades de exploración, indagando si la anterior propuesta es viable en diferentes contextos de población, tanto en niños como jóvenes y en qué medida es o no viable y efectiva. Además, si puede mejorar otras áreas del conocimiento donde se requiera la inferencia, como la estadística entre otros.

El impacto de la abducción en la escuela ha sido significativo. Los rectores de las Instituciones Educativas donde se ha desarrollado esta experiencia, han creído en la propuesta Peirceana y han facilitado el acceso y desarrollo de la misma en los grados $11^{\circ}$ como estrategia vital en la preparación de los estudiantes para las pruebas estatales de educación ICFES, en las cuales se ha evidenciado resultados tangibles.

\section{Referencias}

Barrena, S (2007) “La Razón Creativa" Creci- miento y finalidad del ser humano según C. S. Peirce. Ediciones Rialp, S.A. Madrid Pantoja

"los hábitos y el crecimiento: una perspectiva Peirceana". No 21

Bernárdez, E. "Qué son las lenguas". Alianza. Madrid. 1999

Dorado, P. Carles. Universitat Autónoma de Barcelona. XTEC. 1996

Flavell, John H. "Metacognition and Cognitive Monitoring: A New Area of Cognitive-develomental Inquiry". American Psychologist, Vol 34, Oct 1979

ICFES, 2008,2010; OECD, 2008, 2009. Plan Decenal de Educación 2016-2025, título III El derecho a la educación y la calidad de la educación

Mateos, M. (2009). Aprender a leer textos académicos. Más allá de la lectura reproductiva. En J. I. Pozo Municio y M. P. Pérez Echeverría (Coords.), Psicología del aprendizaje universitario: la formación en competencias. Madrid: ediciones Morata, S.L.

Nubiola, Jaime. "For the methods of thinking that are living activities in men are not objects of reflective consciousness". Charles S. Peirce, CollectedPapers 3.404, 1982. Número 21, 2000.

Nubiola, Jaime. “Walker Percy Y Charles S. Peirce. Abducción Y Lenguaje". Analogía filosófica, México 12/1.1998. P. 87-96.

Vallejo, Antonio (coord.) (2009). Manual básico para la realización de tesinas, tesis y trabajos de investigación. Madrid: editorial EOS.

Zapata, C. (2010). La abducción creativa para mejorar la comprensión textual. Recuperado en file: / / C: / Documents\%20and\%20Settings / XP/Mis\%20documentos/Downloads/IVJornadasArgentinaZapata\%20(1).pdf 